
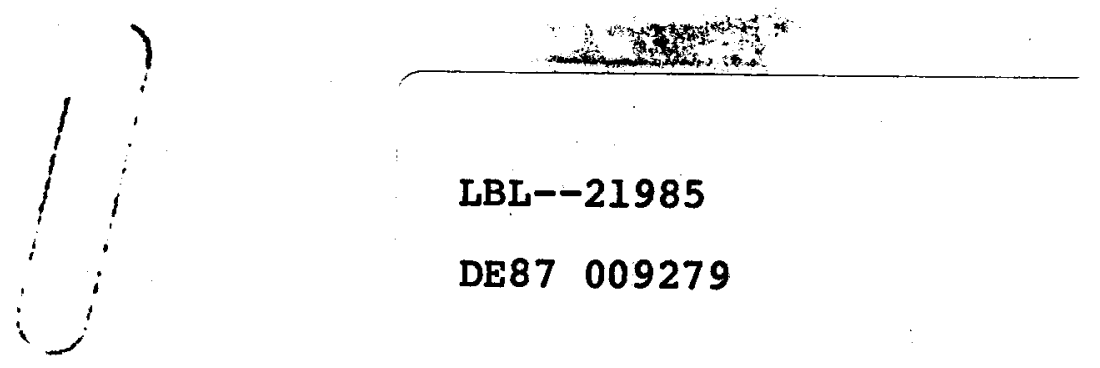

LBL -21985

DE87 009279

\title{
Fracture Detection Using a Grounded Subsurface \\ Vertical Electric Dipole
}

Q. Zhou, ${ }^{*}$ K. H. Lee, ${ }^{*}$ N. E. Goldstein, ${ }^{*}$ H. F. Morrison, ${ }^{*}$ and A. Becker $\dagger$

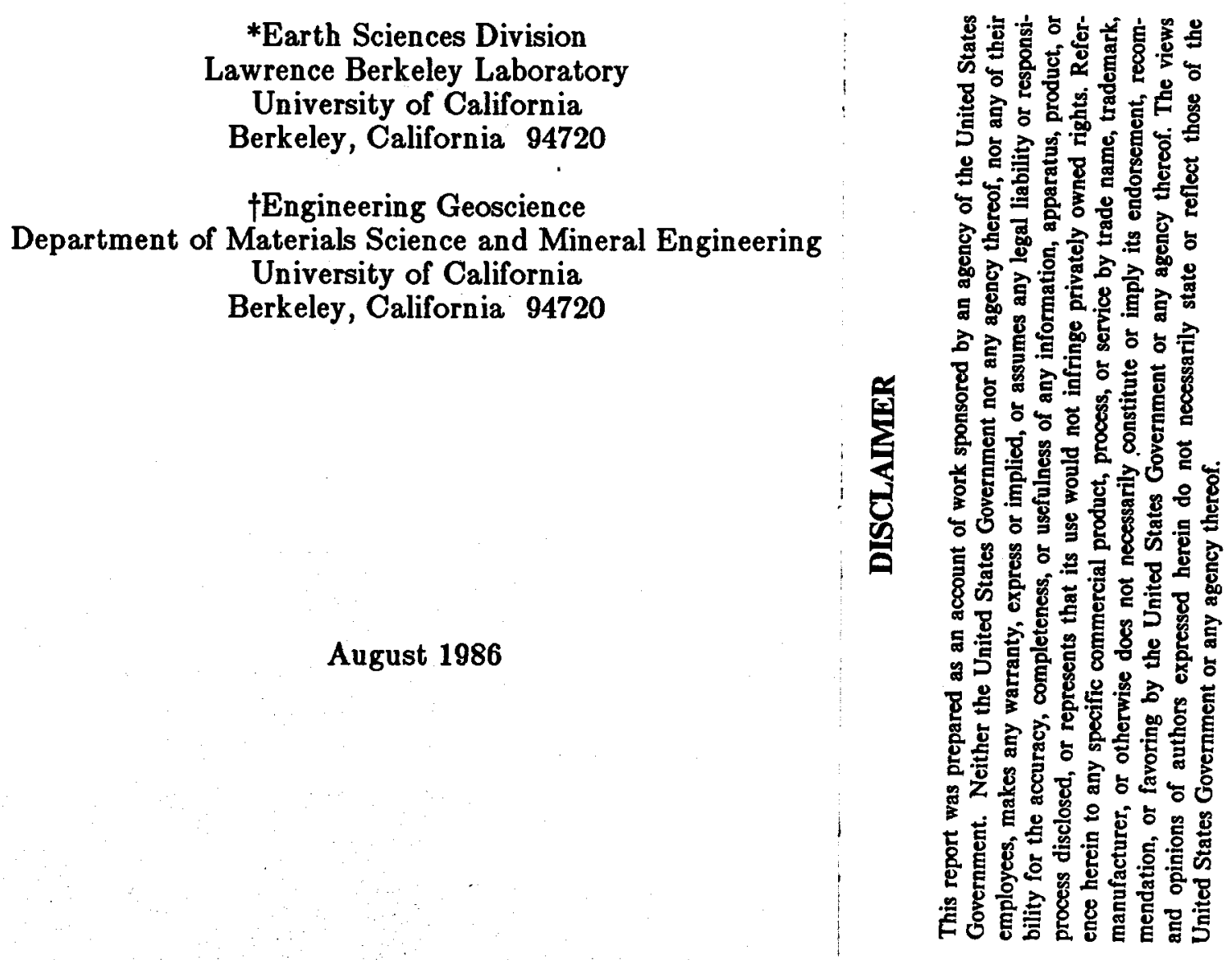

This work was partially supported by the Assistant Secretary for Conservation and Renewable Energy, Office of Renewable Technology, Division of Geothermal and Hydropower Technologies of the U.S. Department of Energy under Contract No. DE-AC0376 SF00098. 


\section{DISCLAIMER}

This report was prepared as an account of work sponsored by an agency of the United States Government. Neither the United States Government nor any agency Thereof, nor any of their employees, makes any warranty, express or implied, or assumes any legal liability or responsibility for the accuracy, completeness, or usefulness of any information, apparatus, product, or process disclosed, or represents that its use would not infringe privately owned rights. Reference herein to any specific commercial product, process, or service by trade name, trademark, manufacturer, or otherwise does not necessarily constitute or imply its endorsement, recommendation, or favoring by the United States Government or any agency thereof. The views and opinions of authors expressed herein do not necessarily state or reflect those of the United States Government or any agency thereof. 


\section{DISCLAIMER}

Portions of this document may be illegible in electronic image products. Images are produced from the best available original document. 


\begin{abstract}
In this paper we study the scattered magnetic field above the surface of the earth due to a buried sheet-like conductor excited by a grounded and oscillating vertical electric dipole (G.V.E.D.) in the earth. The significance of this technique for the detection of water-filled fractures is that there is no magnetic field in the air, assuming that the displacement current is negligible, so long as the G.V.E.D. source is buried in a layered half-space. If any signal is detected it must be due to the presence of a 2-D or 3-D inhomogeneity, such as a sheet-like conductor. Using a numerical modeling approach, we calculated the strength and anomaly shape of the secondary magnetic field from the sheet to determine whether a G.V.E.D. is a suitable source for detecting a major conductive fracture zone.
\end{abstract}

\title{
Introduction
}

A practical problem that arises in geothermal reservoir exploration and development, as well as in other site characterization studies, is to detect and to characterize a major water-filled fracture zone that occurs close to but was missed by a drill hole. In the geothermal case, it is not uncommon to drill a well on the basis of geological information to intersect a specific fluid-producing fault or fracture zone. If the zone is not encountered the questions to be answered are; (1) is the zone locally sealed? or (2) was the well depth, placement and inclination wrong to intersect the permeable zone?

If there is at least one hole available in the area of interest borehole electromagnetic techniques can be used for detecting possible major water-filled fractures (or fracture zones) missed by the holes. The implied assumptions are always that the zone is a good reflector of electromagnetic waves, that the zone is embedded in a less conductive but otherwise homogeneous region, and that in situ thermal and chemical conditions are not so severe that they, would prevent the use of suitably engineered in-hole sources and/or receivers. To date, most of the electromagnetic studies for fracture detection have 
considered a downhole VHF radar source using directional source and receiver antennas in the borehole (Chang et al., 1984). While this technique seems promising, it requires a steerable, directional antenna and a transmitter with sufficient power to offset the high attenuation of the VHF waves in rock, yet the probe must be sufficiently small to operate within the confines of a drillhole.

As part of our on-going study to evaluate numerically the applicability of various electromagnetic techniques for detecting the "missed" fracture zone, we consider in this paper a very simple downhole source consisting of a grounded vertical electric dipole (G.V.E.D.) with observations made at the surface. The fracture zone is approximated by a thin, conductive three-dimensional sheet with variable conductance and dip. The G.V.E.D. is an interesting and important source for this application because in the absence of the thin sheet conductor the magnetic field is everywhere zero on and above the surface of a layered half-space. Numerical calculations have been carried out to compute the horizontal and vertical magnetic field components at the surface over a thin sheet concealed beneath an overburden layer. Calculations were made for variable sheet conductance, sheet dip, overburden thickness and frequency of excitation from $10 \mathrm{~Hz}$ to $10 \mathrm{kHz}$. The purpose of the calculations is to determine whether diagnostic information on the location and dip of the sheet can be extracted from the shapes of the anomaly curves.

\section{Formulation of the Problem}

The physical model studied is shown in Figure 1. The fracture zone is simulated by a rectangular thin sheet with a conductance of $\tau$ siemens (S) located in an otherwise homogeneous, porous, and water-saturated half-space with resistivity $\rho_{2}$. The fractured half-space is concealed by an overburden layer with resistivity $\rho_{1}$ and thickness $D$. The upper edge of the sheet is horizontal and lies at a depth $H(H>D)$ below the surface. The thin sheet is assigned a range of conductances $\tau$ (conductivity-thickness products) 
representative of natural conditions. The G.V.E.D. may be placed anywhere in the earth except very close to the sheet conductor, and has a moment $I \delta l$ that oscillates harmonically at angular frequency $\omega=2 \pi f$.

Other sheet parameters specified in this problem are sheet strike length $L_{\mathrm{a}}$, dip length $L_{\mathrm{b}}$, dip angle $\beta$, and the angle $\gamma$ between sheet strike and the line of observations, or profile line, on the surface. $H_{t x}$ and $H_{\mathrm{rx}}$ are the vertical distances of the transmitter below and the receivers above the ground planes, respectively.

The approach we have used to calculate the scattered (i.e., secondary) magnetic fields at the surface is based on the algorithm developed by Weidelt (1981). In our modified version of the code SHEET we have included a G.V.E.D. source embedded in the conductive medium. A brief description of the algorithm is given here.

Beginning with Maxwell's equations

$$
\begin{gathered}
\nabla \times \mathbf{E}=-i \omega \mu \mathbf{H} \\
\nabla \times \mathbf{H}=(\sigma+i \omega \epsilon) \mathbf{E}+\mathbf{J}
\end{gathered}
$$

Weidelt (1981) derived the following two in tegral equations using the thin sheet approximation (Price, 1949)

$$
\mathrm{E}_{\mathrm{s}}\left(\mathbf{r}_{0}\right)=\mathrm{E}_{\mathrm{ns}}\left(\mathbf{r}_{0}\right)-i \omega \mu \int_{\mathrm{s}} \tau(\mathbf{r}) g\left(\mathbf{r}_{0} / \mathbf{r}\right) \mathrm{E}_{\mathrm{s}}(\mathbf{r}) d s
$$

on the sheet, and

$$
\mathrm{H}\left(\mathbf{r}_{\mathbf{0}}\right)=\mathbf{H}_{\mathbf{n}}\left(\mathbf{r}_{0}\right)+\int_{s} \tau(\mathbf{r}) \mathbf{E}_{\mathbf{s}}(\mathbf{r}) \nabla_{\mathbf{0}} \times g\left(\mathbf{r}_{\mathbf{0}} / \mathbf{r}\right) d s
$$

everywhere. $\mathbf{E}_{\mathrm{s}}$ is the total tangential electric field on the sheet and $\mathbf{E}_{n s}$ is the primary (incident) tangential electric field on the sheet. $H$ is the magnetic field at the point of interest and $H_{n}$ is the primary magnetic field. Throughout the analysis $\mu$ is set equal to the free space permeability $\mu_{0}$, and $g\left(r_{0} / r\right)$ is the Green's dyadic function relating the tangential current distribution on the sheet with the electric fields everywhere. 
To modify Weidelt's SHEET program for a G.V.E.D. source buried in the conductive medium we only have to change the primary tangential field $\mathbf{E}_{n s}$ on the sheet. The primary magnetic field $H_{n}$ in the air will be zero assuming that the displacement current is negligible. The derivation of $\mathbf{E}_{n s}$ due to a G.V.E.D. source is given in Appendix A.

\section{Numerical Results and Analysis}

A few numerical checks have been made to ensure the desired performance of the algorithm. The primary electric fields at low frequencies have been found to correctly asymptote to DC fields inside the conductive medium. Another numerical check has been made by setting the overburden resistivity equal to that of the host rock resistivity, and then making the computation as if the earth were two-layered. Keeping the source position fixed, we also varied the thickness of the overburden, which is equivalent to placing the source in the overburden or in the host rock. The results obtained for different thicknesses of the overburden have been found to be the same, which shows that the program is at least self-consistent. Other checks, such as reciprocity, duality and symmetry, also show that the results are acceptable.

Some typical model results have been obtained to demonstrate the characteristics of the anomalous fields from the G.V.E.D. and its advantages over the vertical magnetic dipole (V.M.D.) source. The model parameters held fixed in this study are listed in Table 1. The center of the top edge of the sheet $\left(x_{c}, y_{c}\right)$ is located directly below the line of observations (or profile line), and the profile line coincides with the $x$-axis for these calculations. In the following cases we allow parameters $\tau, f, \beta$ and $\rho_{1}$, to vary, changing only one at a time to find its effect on the anomaly.

The first model considered is a vertical $\left(\beta=90^{\circ}\right)$ sheet in a uniform half-space, $\rho_{1}=\rho_{2}=100$ ohm-m, with varying conductance; $\tau=0.5,1,3,5$ and $10 \mathrm{~S}$. The real and imaginary components of the horizontal magnetic field $\left(H_{y}\right)$ at $1000 \mathrm{~Hz}$ are plotted in Figure 2.1. Contrary to what one might expect, the real component decreases with 
Table 1

Fixed Model Parameters Used in the G.V.E.D. Study

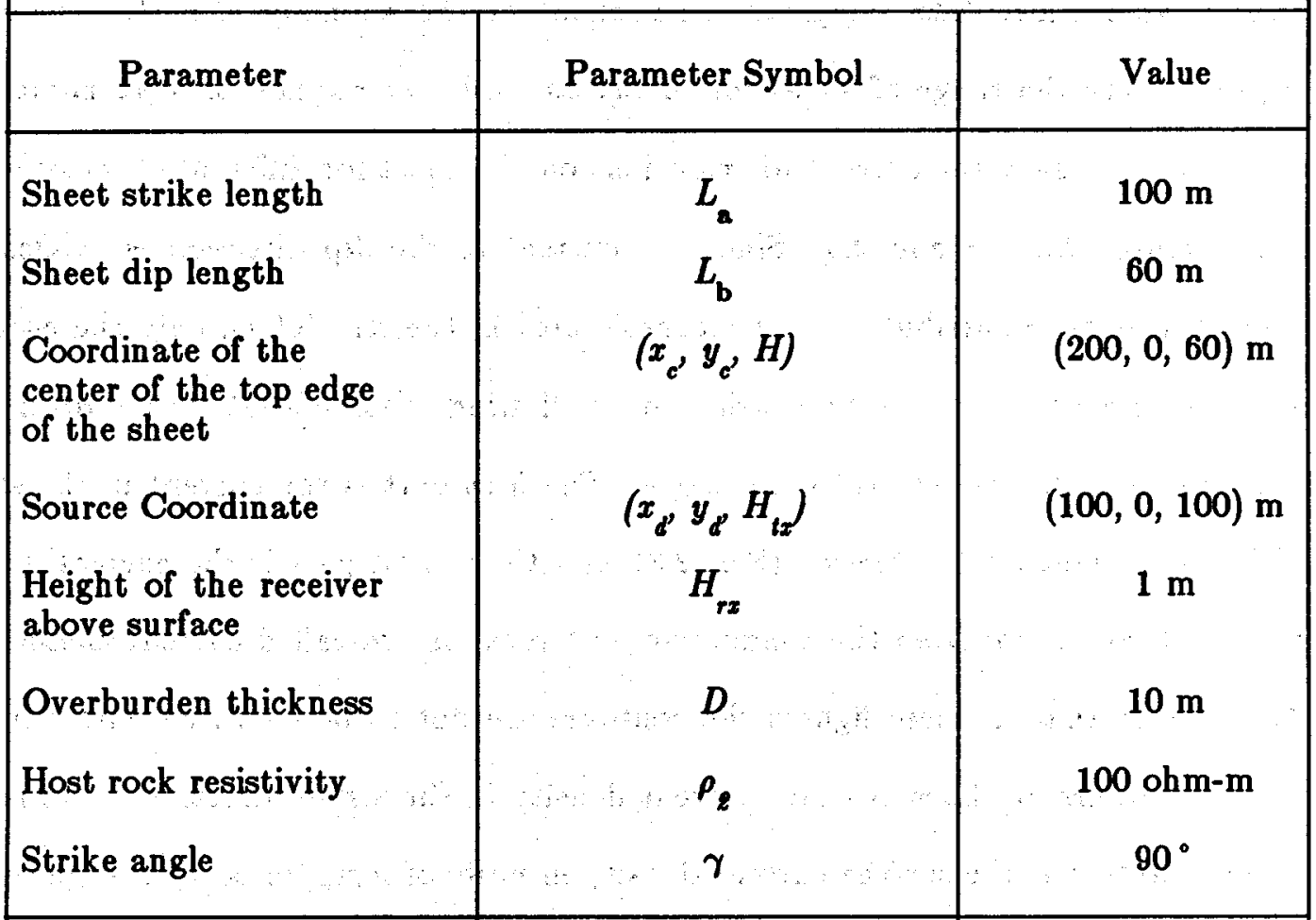


increasing sheet conductance from 0.5 to $3 \mathrm{~S}$ and crosses zero between 3 and $5 \mathrm{~S}$. However, as the conductance increases further, the real component of $H_{y}$ changes sign and increases in amplitude. The imaginary component of the anomaly is better behaved, varying little over the range of sheet conductances used. To explain this phenomenon, we calculated and show the current distributions on the sheet for different sheet conductances in Figures 2.2, 2.3 and 2.4. Since the current in the dip direction is vertical in this case, it does not contribute to the magnetic field in the air. Thus, only the current in the strike direction needs to be considered. Following Weidelt's (1981) approach the total current (Fig. 2.2) is split in to two parts. The first part is the current in the sheet caused by electromagnetic induction (Fig. 2.3), and the second part is the current drawn in to the sheet conductor from the conductive host rock, the so-called current channeling (Fig. 2.4). Note that in these figures the contours are not to be confused with current lines. The contours are lines of equal current density in the strike direction. The labeling on the contours is the surface current density in units of Amp $/ \mathrm{m} \times 10^{7}$. Figures 2.3 and 2.4 show that both parts of the current increase in amplitude as the sheet conductance $r$ increases. For the real component of the total current, the current channeling is dominant when $\tau$ is small. As $\tau$ increases the induced current on the sheet increases and finally dominates. Since the two parts of the current flow in opposite directions on the upper edge of the sheet conductor, the real component of the total current on the upper part of the sheet in Figure 2.2 changes sign as $\tau$ increases. This explains why the real component of the magnetic field $\left(H_{y}\right)$ changes sign as $\tau$ increases. As for the imaginary component of the current, the two parts of the current are in the same direction on the upper part of the sheet, and therefore there is no sign change for the imaginary component of the magnetic field as $\tau$ increases.

Figure 3 shows magnetic field $\left(H_{y}\right)$ anomaly at different frequencies. The model is a vertical thin sheet $(\tau=1.0 \mathrm{~S})$ buried in a uniform half-space; $\rho_{1}=\rho_{2}=100 \mathrm{ohm}-\mathrm{m}$. Both the real and imaginary components of the responses are plotted for varying 
frequencies of $10,100,1000,3000$, and $10000 \mathrm{~Hz}$. The effect of the increasing frequency on the observed magnetic field anomaly is essentially similar to that of the increasing conductance of the sheet. At low frequencies the real component is dominant, but with the increase of the frequency the imaginary component becomes larger. The responses for the real component at 10 and $100 \mathrm{~Hz}$ are essentially equal and cannot be differentiated in the figure. At higher frequencies, the responses of the real component change sign as in the case of increasing sheet conductance. For a G.V.E.D. source there always exists current channeling and its associated magnetic fields even if the frequency goes to zero because of the conduction current from the source. On the other hand, for a magnetic dipole source both the real and imaginary components of the secondary field would vanish as the frequency goes to zero.

The next model studied is the same as the previous one, except that the resistivity of the 10-m-thick overburden is varied and the frequency used is held at $1000 \mathrm{~Hz}$. The four curves in Figure 4 are for overburden resistivity $\left(\rho_{1}\right)$ of $1,3,10$ and $100 \mathrm{ohm}-\mathrm{m}$. It was found, as expected, that the amplitude of the anomaly, both the real and imaginary components, decreases as the overburden resistivity decreases. At lower values of $\rho_{1}$, more conduction current from the source and more of the current induced in the host rock would flow into the overburden, resulting in less current channelled in to the sheet. For $\rho_{1}=1$ and $3 \mathrm{ohm}-\mathrm{m}$, for example, the current channelled into the sheet is insignificant, and consequently the anomaly decreases rapidly. The induced current within the sheet seems to have contributed most of the anomaly, if any, in this case.

One of the important parameters involving sheet response study is the dip angle $(\beta)$. The plots in Figure 5 show the magnetic field anomaly for a dipping sheet with $\beta$ $=90^{\circ}, 75^{\circ}, 60^{\circ}, 45^{\circ}$ and $30^{\circ}$. The model is a thin sheet of conductance $1.0 \mathrm{~S}$, buried in a uniform half-space of $100 \mathrm{ohm}-\mathrm{m}$. The frequency used is again fixed at $1000 \mathrm{~Hz}$. Major changes in the shape of the anomaly occur as the sheet dips away from the vertical position; the zero crossing disappears, and the anomaly sharply increases with a 
sudden phase reversal at smaller dip angle $\left(\beta=30^{\circ}\right.$ in this case). The increase in anomaly is caused by the fact that, as the bottom edge of the sheet swings clockwise from the vertical position, the sheet comes closer to the source and to the surface. In addition, the current in the dip direction is no longer vertical in this case, and therefore, it contributes increasingly more to the magnetic fields as the dip angle decreases. The abrupt phase reversal of the anomaly is directly related to the position of the G.V.E.D. source with respect to the plane that contains the sheet. The current in the sheet, especially the conduction current, reverses its direction depending upon the relative position of the source to the sheet plane, which in turn results in the observed phase reversal in the magnetic field. If a sufficient number and density of surface measurements are made with respect to one or more source positions, we may then be able to deduce some of the important sheet geometrical parameters, such as its strike length, strike angle, and dip angle.

Finally, a comparison has been made between the anomalous magnetic fields from the sheet conductor due to a buried V.M.D. (Zhou and Becker, 1985) and those due to a G.V.E.D. excitation. Figures 6.1, 6.2 and 6.3 are magnetic fields $H_{x}, H_{y}$ and $H_{z}$, respectively, from the G.V.E.D. source, and Figures 6.4 and 6.5 are magnetic fields $H_{z}$ and $H_{z}$ from the V.M.D. source. The model is a vertical sheet of conductance $1.0 \mathrm{~S}$, buried in a uniform half-space of resistivity $100 \mathrm{ohm}-\mathrm{m}$. The frequency used is $1000 \mathrm{~Hz}$ for both source types. The results show that the anomalies associated with the G.V.E.D. source are more complex in shape, suggesting that the G.V.E.D. anomalies are more diagnostic of fracture parameters. Based on this and the fact that the anomalies associated with the V.M.D. source is superimposed on the large primary fields, the technique involving a G.V.E.D. source seems more attractive and useful for the detection of a major fracture zone. 


\section{Conclusions}

In our evaluation of surface-to-borehole electromagnetic methods for detecting a major fracture zone in a geothermal reservoir or other related geological situations, we have analyzed and presented a numerical study involving the use of the G.V.E.D. source in the frequency domain. Particular attentions have been paid to the effect of the changing conductance of the thin sheet that simulate a conductive fracture. The effect of the changing frequency is found similar to that of the changing conductance, as long as the product of the conductance and the frequency remains small.

The anomalous currents contained in a vertical sheet conductor generate surface magnetic fields whose profile is either symmetric or antisymmetric in shape. This is a valuable diagnostic information in finding a vertical fracture, since no other 2-D or 3-D lateral inhomogeneities can produce such an anomaly when a fixed source is used. In the case of a dipping fracture, including a horizontal one, the symmetry in the anomaly disappears and, without further comprehensive analysis of the subject, it cannot be said whether the shape of the anomaly is distinguishable from those caused by other types of inhomogeneites. Within the context of models studied in this paper, however, the dip angle and the location of the down-dip portion of the sheet with respect to the G.V.E.D. source may be approximately deduced by examining the phase and the amplitude of the anomalous magnetic field on the surface.

From the study we also find that the G.V.E.D. source has certain advantages over the V.M.D. source. The main reason is that the magnetic field measured in the air due to a G.V.E.D. is only caused by the presence of a lateral inhomogeneity. Also, with a conductor present signal is more diagnostic than from a V.M.D. excitation. Therefore, one can safely assume that there is a better chance of detecting a conductive target if the G.V.E.D. is used. Because the anomalies due to the G.V.E.D. excitation are more complex, care should be taken when one attemps to in terpret G.V.E.D. field data. 


\section{Acknowledgements}

This work was partially supported by the U.S. Department of Energy, Office of Renewable Technologies, Geothermal Technology Division under Contract No. DEAC03-76SF00098. We wish to express our thanks to Ms. M. Mackiewicz for providing some numerical examples used in testing the program. 


\section{References}

Chang, H-T., Suhler, S.A., and Owen, T.E., 1984, Evaluation of borehole electromagnetic and seismic detection of fractures: Sandia National Laboratories, Report SAND 84-7109, 75 p.

Price, A. T., 1949, The induction of electric currents in nonuniform thin sheets and shells: Q. J. Mech. Appl. Math., v. 2, p. 283-310.

Wait, J. R., 1982, Geo-electromagnetism: Academic Press, Inc., p. 140-146.

Weaver, J. T., 1967, The quasi-static field of an electric dipole embedded in a two-layer conducting half-space: Canadian J. Physics, v. 45, p. 1981-2002.

Weidelt, P., 1981, Report on Dipole Induction by a Thin Plate in a Conductive Halfspace with an Overburden: Federal Institute for Earth Science and Raw Materials, Hannover, Germany.

Zhou, Q. and Becker, A., 1985, Distortion of electromagnetic waves by geological structure: Engineering Geoscience, Univ. of California, Berkeley, Interim report submitted to Electronic Engineering Department of Lawrence Livermore National Laboratory. 


\section{Appendix A}

The electromagnetic field from a G.V.E.D. embedded in a two layered half space is evaluated. Using a vector potential $A$ and a scalar potential $V$ we write

$$
\begin{gathered}
\mathbf{H}=\nabla \times \mathbf{A} \\
\mathbf{E}=-i \omega \mu \mathbf{A}-\nabla \boldsymbol{V} .
\end{gathered}
$$

If we choose

$$
V=-\frac{1}{\sigma+i \omega \epsilon} \nabla \cdot \mathbf{A}
$$

Then, from equations (1) and (2)

$$
\left(\nabla^{2}-\gamma^{2}\right) \mathbf{A}=-\mathbf{J}
$$

where

$$
\gamma^{2}=i \omega \mu(\sigma+i \omega \epsilon)
$$

If the medium is both homogeneous and unbounded

$$
\begin{gathered}
A=\frac{1}{4 \pi} \iint_{-\infty}^{+\infty} \int \mathrm{J}\left(x^{\prime}, y^{\prime}, z^{\prime}\right) \frac{e^{-\gamma r}}{r} d x^{\prime} d y^{\prime} d z^{\prime} \\
r^{2}=\left(x-x^{\prime}\right)^{2}+\left(y-y^{\prime}\right)^{2}+\left(z-z^{\prime}\right)^{2}
\end{gathered}
$$

In the presence of a horizontal boundary, the vector potential $\mathbf{A}$ must have a secondary component in the direction normal to the boundary. In the cylindrical coordinate as shown in Figure A.1, a G.V.E.D. in a horizontally stratified homogeneous medium has only the $z$ component vector potential

$$
\mathbf{A}=A_{2}(\rho, z) \mathbf{z}
$$

then the equations (A.1) and (A.2) become

$$
H=-\frac{\partial A_{\Sigma}}{\partial \rho}
$$




$$
\mathrm{E}=\frac{i \omega \mu}{\gamma^{2}} \frac{\partial^{2} A_{\mathrm{z}}}{\partial \rho \partial z} \rho+i \omega \mu\left(\frac{1}{\gamma^{2}} \frac{\partial^{2} A_{\mathrm{z}}}{\partial z^{2}}-A_{\mathrm{z}}\right) \mathrm{z}
$$

The small bold characters $\rho$ and $z$ represent unit vectors in $\rho$ and $z$ directions, respectively. The boundary conditions at layer boundaries are

$$
A_{\mathrm{z}}^{-}=A_{\mathrm{z}}^{+} ; \quad \frac{1}{\gamma_{-}^{2}} \frac{\partial A_{\mathrm{z}}^{-}}{\partial z}=\frac{1}{\gamma_{+}^{2}} \frac{\partial A_{\mathrm{z}}^{+}}{\partial z}
$$

and the "+" and "-" signs denote the different sides of the boundary.

Let the depth to the source be $H_{t x}$ and the thickness of the overburden be $D$. The G.V.E.D. source is driven by a current $I$ from $C_{1}$ to $C_{2}$ then through the medium back to $C_{1}$ (Fig. A.1). The separation between $C_{1}$ and $C_{2}$ is $\delta l$ and thus the electric dipole moment $p=I \delta l$.

(1) Homogeneous and unbounded whole space.

$$
A_{2}=\frac{p}{4 \pi r} e^{-\gamma r}=\frac{p}{4 \pi} \int_{0}^{\infty} \frac{\gamma}{u} e^{-u\left|z-H_{\mathrm{tx}}\right|} J_{0}(\lambda \rho) d \lambda
$$

where $u^{2}=\lambda^{2}+\gamma^{2}$.

(2) Half-space with the source in the lower half-space.

$$
\begin{aligned}
& A_{z 0}=\frac{p}{4 \pi} \int_{0}^{\infty} A_{1}(\lambda) e^{u_{0} z} J_{0}(\lambda \rho) d \lambda \\
& A_{21}=\frac{p}{4 \pi} \frac{e^{-\gamma_{1} r}}{r}+\frac{p}{4 \pi} \int_{0}^{\infty} \frac{\lambda}{u_{1}} B_{1}(\lambda) e^{-u_{1}\left(z+H_{t x}\right)} J_{0}(\lambda \rho) d \lambda
\end{aligned}
$$

where

$$
\begin{aligned}
& A_{1}(\lambda)=\frac{\lambda}{u_{1}}\left(1+R_{1}\right) e^{-u_{1} H_{i x}} \\
& B_{1}(\lambda)=R_{1} \\
& u_{j}^{2}=\lambda^{2}+\gamma_{j}^{2} ; j=0,1 ; j=0 \text { is for air and } j=1 \text { for the lower half-space. }
\end{aligned}
$$


(3) Half-space with an overburden; source in the lower half-space.

$$
\begin{aligned}
& A_{20}=\frac{p}{4 \pi} \int_{0}^{\infty} A_{2}(\lambda) e^{u_{0} \sigma^{z}} J_{0}(\lambda \rho) d \lambda \\
& A_{22}=\frac{p}{4 \pi} \frac{e^{-\gamma_{2} r}}{r}+\frac{p}{4 \pi} \int_{0}^{\infty} \frac{\lambda}{u_{2}} B_{2}(\lambda) e^{-u_{2}\left(z+H_{\mathrm{tx}}\right)} J_{0}(\lambda \rho) d \lambda
\end{aligned}
$$

where

$$
\begin{aligned}
& A_{2}(\lambda)=\frac{\frac{\lambda}{u_{2}}\left(1+R_{1}\right)\left(1+R_{2}\right) e^{-u_{1} D} e^{-u_{2}\left(H_{t x}-D\right)}}{1+R_{1} R_{2} e^{-2 u_{1} D}} \\
& B_{2}(\lambda)=\frac{\left(R_{2}+R_{1} e^{-2 u_{1} D}\right) e^{2 u_{2} D}}{1+R_{1} R_{2} e^{-2 u_{1} D}} \\
& u_{j}^{2}=\lambda^{2}+\gamma_{j}^{2} ; j=0,1,2 ; j=0 \text { is for air; } j \stackrel{\text { I }}{=} 1 \text { for overburden and } \\
& j=2 \text { for lower half-space. }
\end{aligned}
$$

(4) Half-space with an overburden; source in the overburden.

$$
\begin{aligned}
& A_{\mathrm{z} 0}=\frac{p}{4 \pi} \int_{0}^{\infty} A_{3}(\lambda) e^{u_{0}{ }^{z}} J_{0}(\lambda \rho) d \lambda \\
& A_{\mathrm{z} 2}=\frac{p}{4 \pi} \int_{0}^{\infty} \frac{\lambda}{u_{2}} B_{3}(\lambda) e^{-u_{2}\left(z+H_{\mathrm{ux}}\right)} J_{0}(\lambda \rho) d \lambda
\end{aligned}
$$

where

$$
\begin{aligned}
& A_{3}(\lambda)=\frac{\frac{\lambda}{u_{1}}\left(1+R_{1}\right)\left(1-R_{2} e^{-2 u_{1}\left(D-H_{t x}\right)}\right) e^{-u_{1} H_{\mathrm{tx}}}}{1+R_{1} R_{2} e^{-2 u_{1} D}} \\
& B_{3}(\lambda)=\frac{\frac{u_{2}}{u_{1}}\left(1-R_{2}\right)\left(1+R_{1} e^{-2 u_{1} H_{\mathrm{tx}}}\right)}{1+R_{1} R_{2} e^{-2 u_{1} D}} e^{u_{2}\left(D+H_{\mathrm{tx}}\right)} e^{-u_{1}\left(D-H_{\mathrm{tx}}\right)}
\end{aligned}
$$

and the reflection coefficients, $R_{1}$ and $R_{2}$, used throughout are given as 


$$
R_{1}=\frac{u_{1} \gamma_{0}^{2}-u_{0} \gamma_{1}^{2}}{u_{1} \gamma_{0}^{2}+u_{0} \gamma_{1}^{2}} ; \quad R_{2}=\frac{u_{2} \gamma_{1}^{2}-u_{1} \gamma_{2}^{2}}{u_{2} \gamma_{1}^{2}+u_{1} \gamma_{2}^{2}}
$$

Since the displacement current is negligible and the air conductivity is zero, we have $\gamma_{0} \equiv 0$. Thus from the expressions it is found that $A_{i}(\lambda) \equiv 0, i=1 \sim 3$, and consequently $A_{20} \equiv 0$. This confirms that there is no field in the air when a G.V.E.D. is used in a layered half-space.

Using the equations (A.6) and (A.7), we can compute the electric field in the lower half-space. After computing $E$, and $E_{z}$ of the two layered earth, the primary tangential fields $E_{n a}\left(r_{0}\right)$ in a (parallel to the strike) and $b$ (parallel to the dip) directions on the sheet can be easily obtained.

$$
\begin{gathered}
E_{\mathrm{a}}=E_{\mathrm{\rho}}(\cos \theta \cos \gamma+\sin \gamma \sin \theta) \\
E_{\mathrm{b}}=E_{\rho}(-\sin \gamma \cos \beta \cos \theta+\cos \gamma \cos \beta \sin \theta)+E_{\mathrm{z}} \sin \beta
\end{gathered}
$$

where

$$
\cos \theta=\frac{\mathbf{x}}{\sqrt{x^{2}+y^{2}}} ; \quad \sin \theta=\frac{y}{\sqrt{x^{2}+y^{2}}}
$$

$\beta$ is the dip angle of the sheet and $\gamma$ is the angle between the sheet strike and the profile line. $x, y$, and $\theta$ are shown in Figure A.1. 
(A) 3-D View

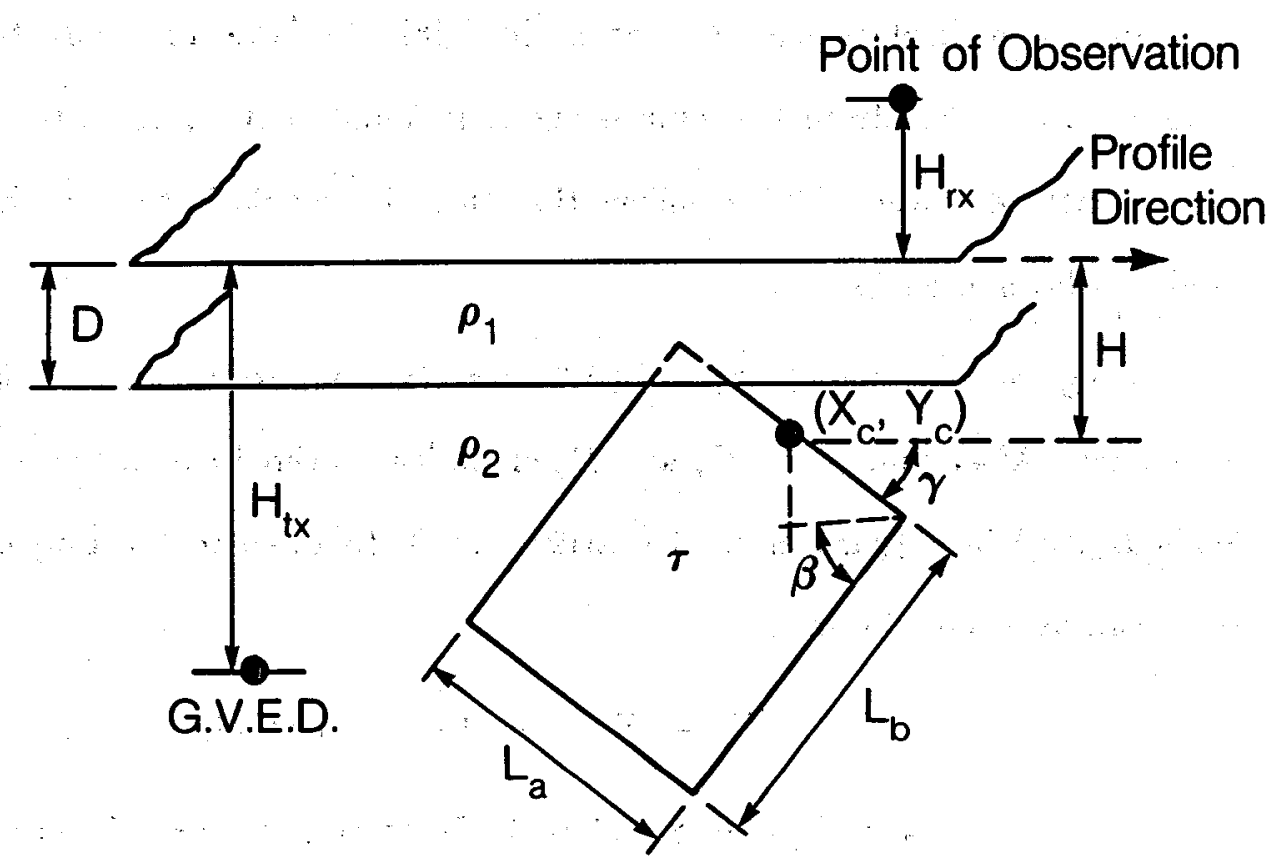

(B) Top View

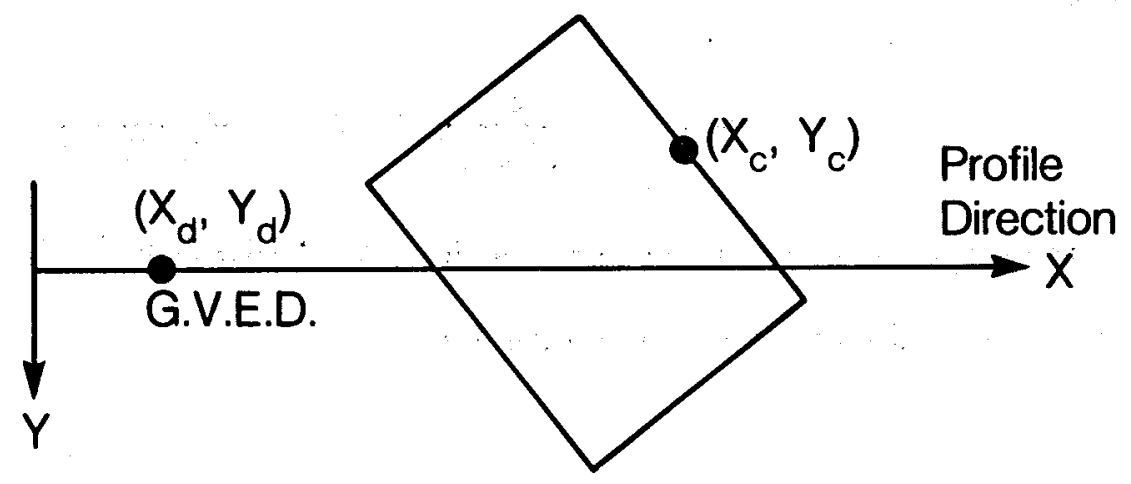

XBL 869-10985

Figure 1. A conductive thin sheet buried in a conductive half-space (resistivity $\rho_{2}$ ) with an overburden (resistivity $\rho_{1}$ ) of thickness $D$. The rectangular thin sheet is characterized by its conductance $(\tau)$, strike length $\left(\mathbf{L}_{\mathbf{a}}\right)$, dip extent $\left(\mathbf{L}_{b}\right)$, dip angle $(\beta)$, and strike $(\gamma)$. The coordinate of the center of the top edge of the sheet is $\left(x_{c}, y_{c}, H\right)$. The medium is excited by a grounded vertical electric dipole located at $\left(x_{d}, y_{d}, H_{t x}\right)$, and the resultant magnetic field is measured at $\left(x, 0, H_{r x}\right)$ on or above the ground. 

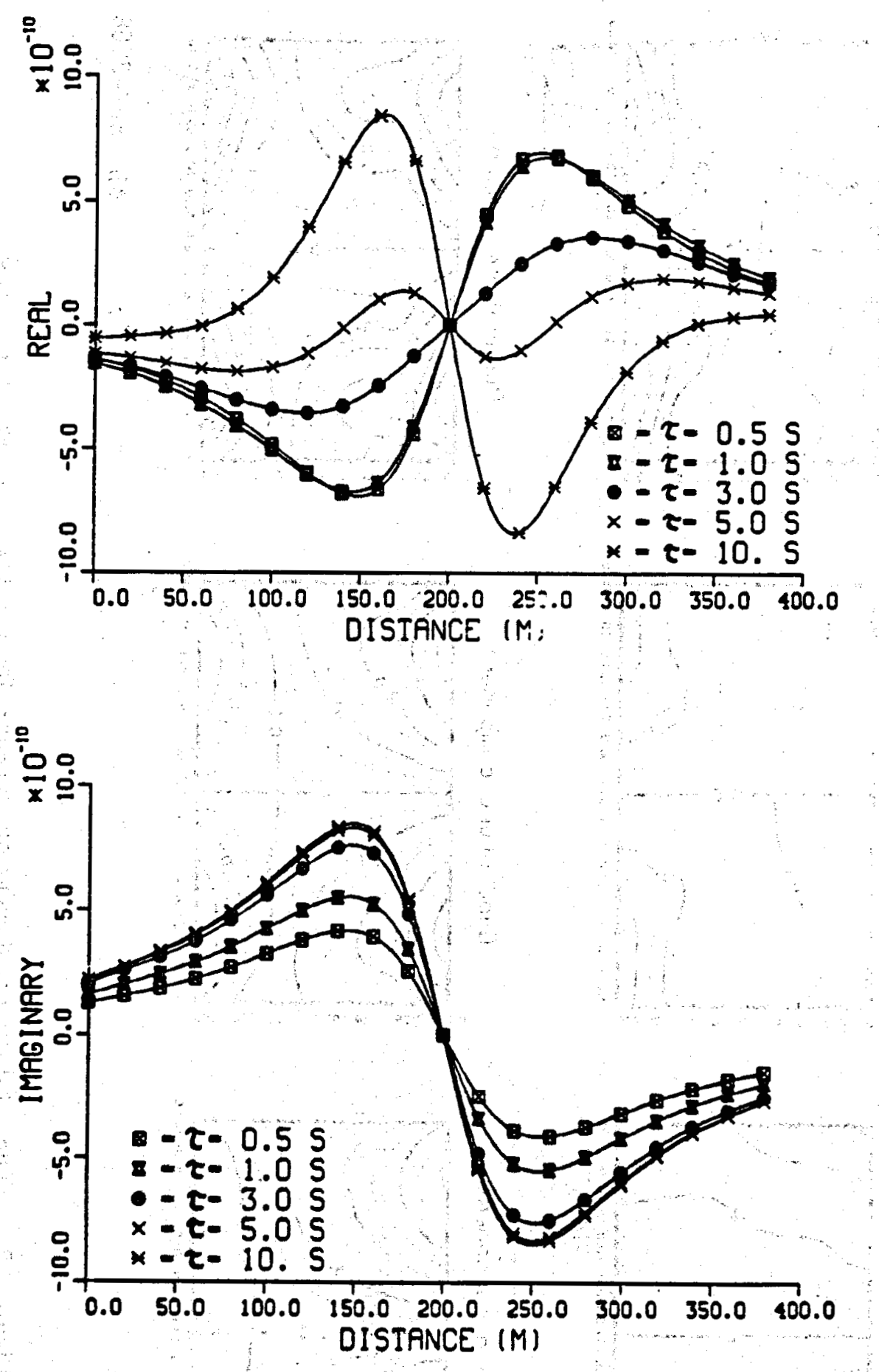

XBL $869-3567$

Figure 2.1. The magnetic field $\left(H_{y}\right)$ anomaly for varying sheet conductance; $\tau=0.5$, $1,3,5,10 \mathrm{~S}$. The sheet is vertical $\left(\beta=90^{\circ}\right)$, and is buried in a uniform half-space of resistivity $\rho_{1}=\rho_{2}=100 \mathrm{ohm}-\mathrm{m}$. The frequency used is $1000 \mathrm{~Hz}$. The magnetic field intensity is expressed in $\mathrm{A} / \mathrm{m} \times 10^{7}$ (SI units). 
$\tau=1 \mathrm{~S}$

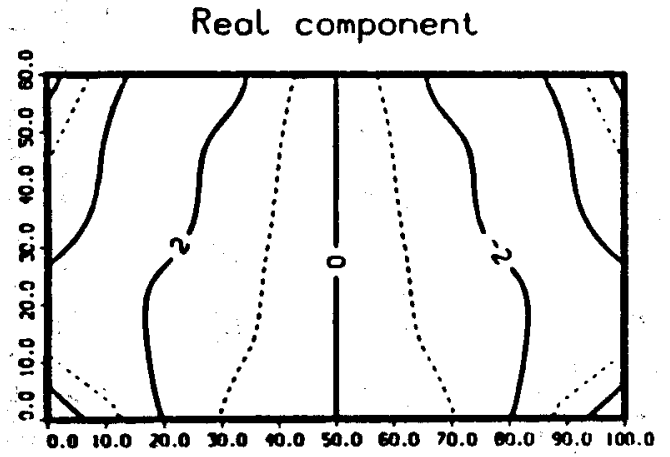

Ouadrature componerit

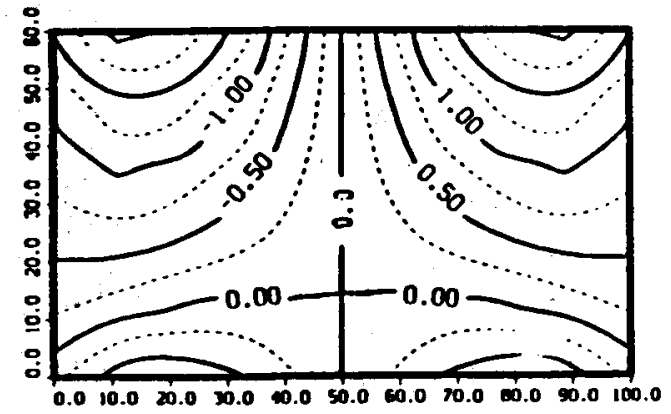

$\tau=5 \mathrm{~S}$

Real componenl

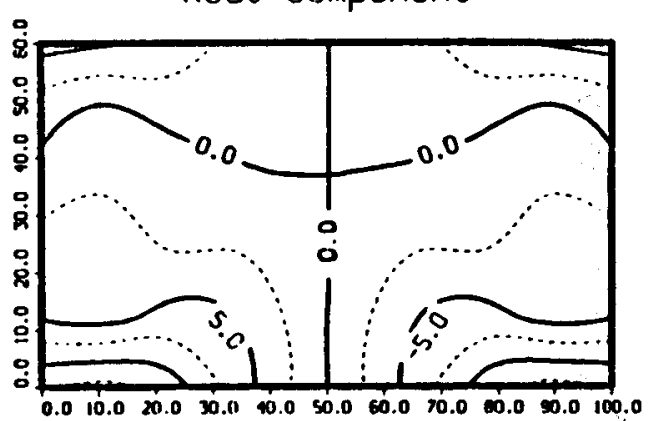

Ouadrature component

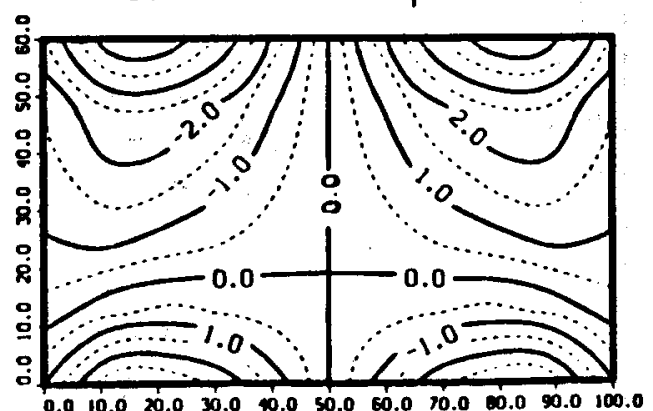

$\tau=10 \mathrm{~S}$

Real component

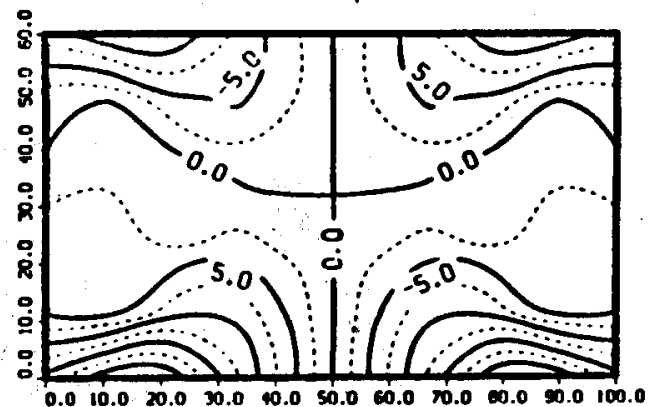

Quadralure component

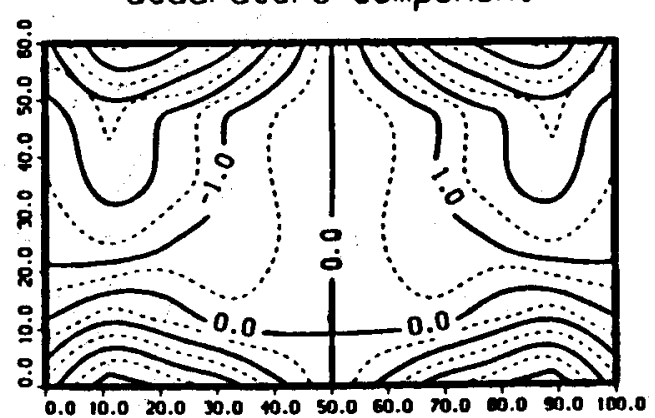

XBL $869-3568$

Figure 2.2. The total current density on the sheet for varying conductance; $\tau=1,5$, $10 \mathrm{~S}$. The con tour represents equal current density in the strike direction. The sheet is vertical $\left(\beta=90^{\circ}\right)$, and is buried in a uniform half-space of resistivity $\rho_{1}=\rho_{2}=100 \mathrm{ohm}-\mathrm{m}$. The frequency used is $1000 \mathrm{~Hz}$. The magnetic field intensity is expressed in $\mathrm{A} / \mathrm{m} \times 10^{7}$ (SI units). 
$\tau=1 \mathrm{~S}$

Reol component

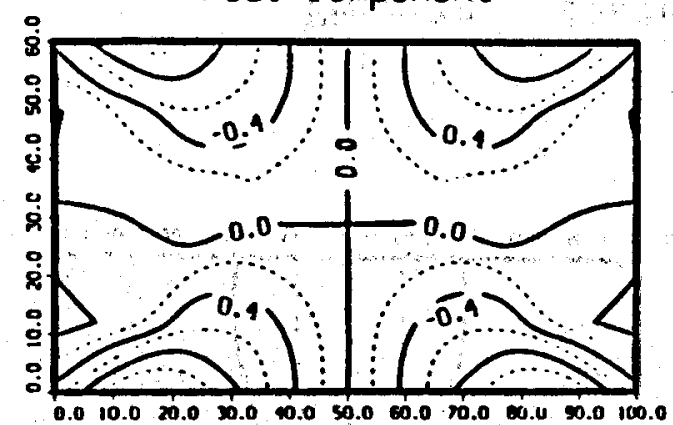

Duodroture companent

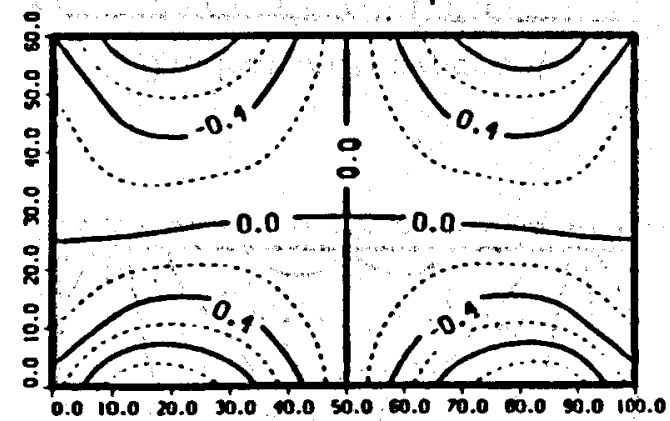

$\tau=5 \mathrm{~S}$

Real component

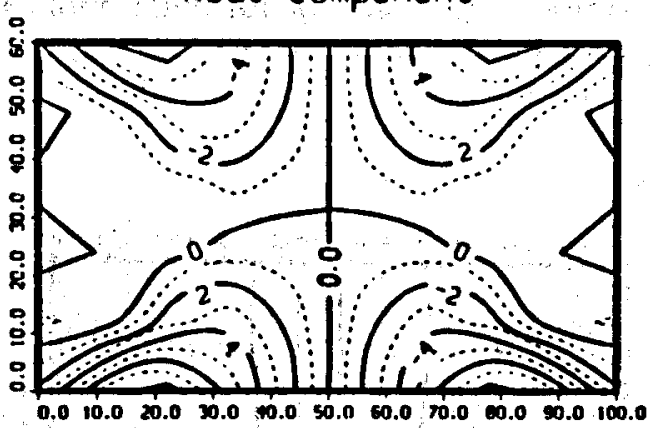

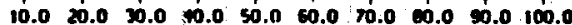
it

Ouodral ure component

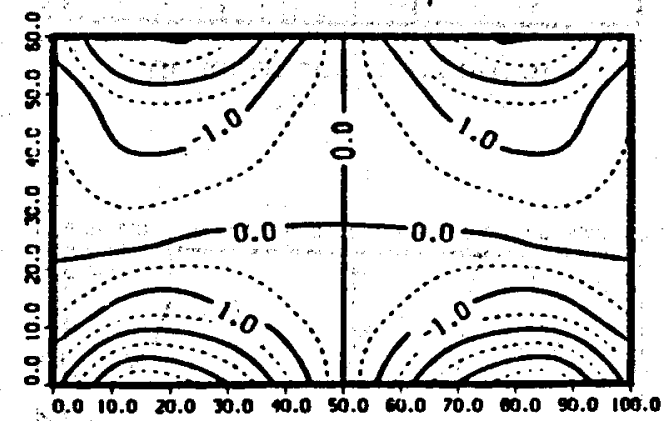

$\tau=10 \mathrm{~S}$

Reul component

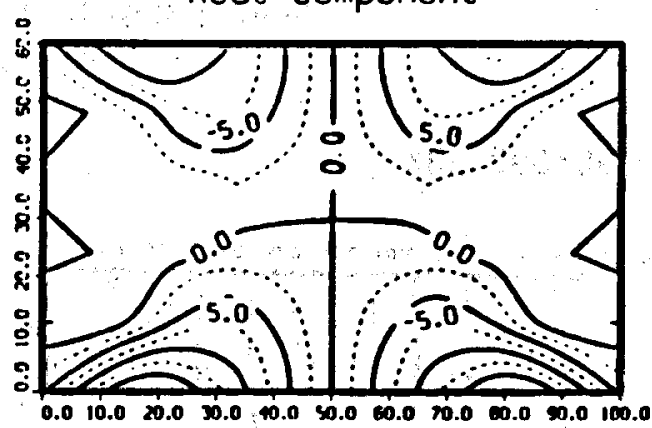

Ouadrature component

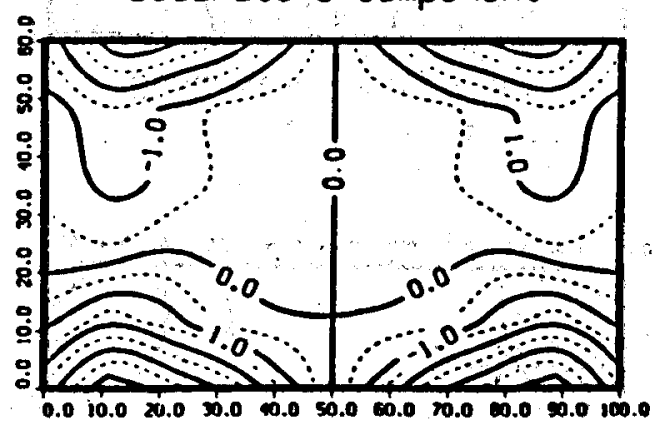

XBL 869-3569

Figure 2.3. The induced current that circulates and closes on the sheet for varying conductance; $\tau=1,5,10 \mathrm{~S}$. The contour represents equal current density in the strike direction. The sheet is vertical $\left(\beta=90^{\circ}\right)$, and is buried in a uniform half-space of resistivity $\rho_{1}=\rho_{2}=100 \mathrm{ohm}-\mathrm{m}$. The frequency used is $1000 \mathrm{~Hz}$. The magnetic field intensity is expressed in $\mathrm{A} / \mathrm{m}$ $\times 10^{7}$ (SI units). 
$\tau=1 \mathrm{~S}$

Reol component

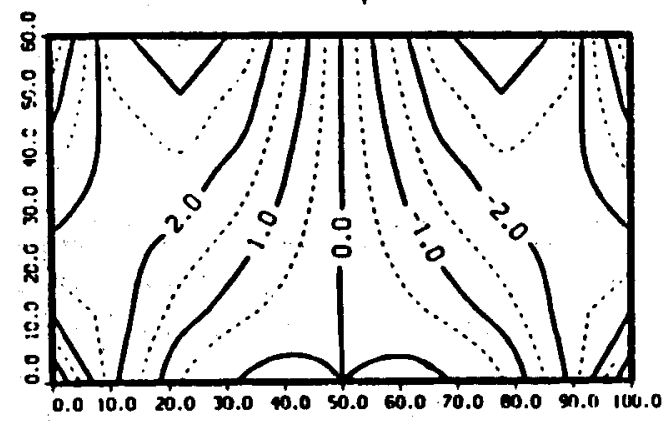

Ouadroture component

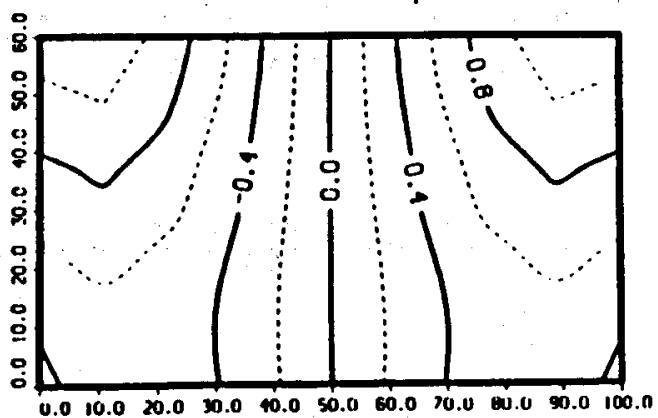

$\tau=5 \mathrm{~S}$

Reol componerit

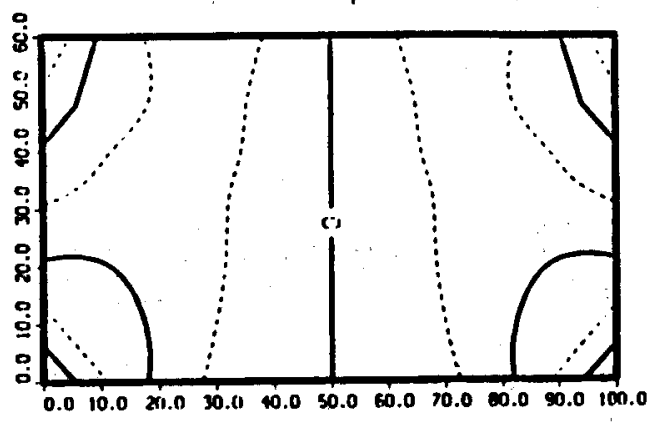

Duadrature component

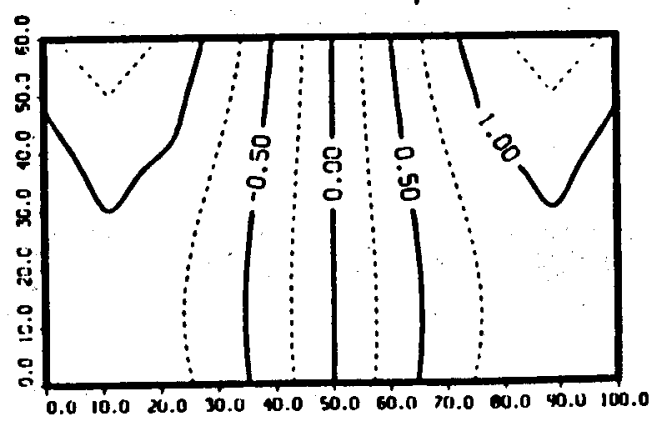

$\tau=10 \mathrm{~S}$

Real component

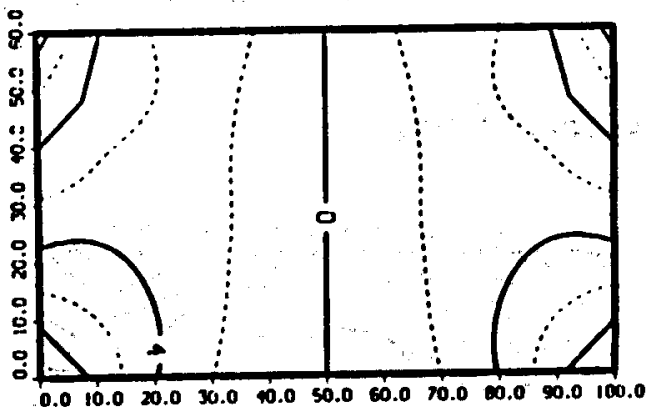

Ouadralure component

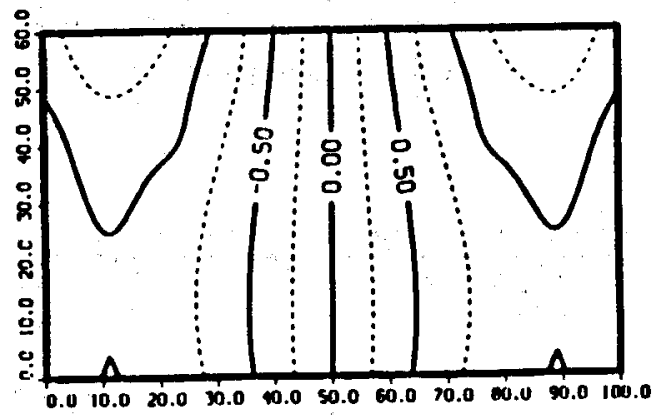

XBL $869-3570$

Figure 2.4. The current channeling for varying conductance; $\tau=1,5,10 \mathrm{~S}$. The contour represents equal current density in the strike direction. The sheet is vertical $\left(\beta=90^{\circ}\right)$, and is buried in a uniform half-space of resistivity $\rho_{1}=\rho_{2}=100$ ohm-m. The frequency used is $1000 \mathrm{~Hz}$. The magnetic field intensity is expressed in $\mathrm{A} / \mathrm{m} \times 10^{7}$ (SI units). 

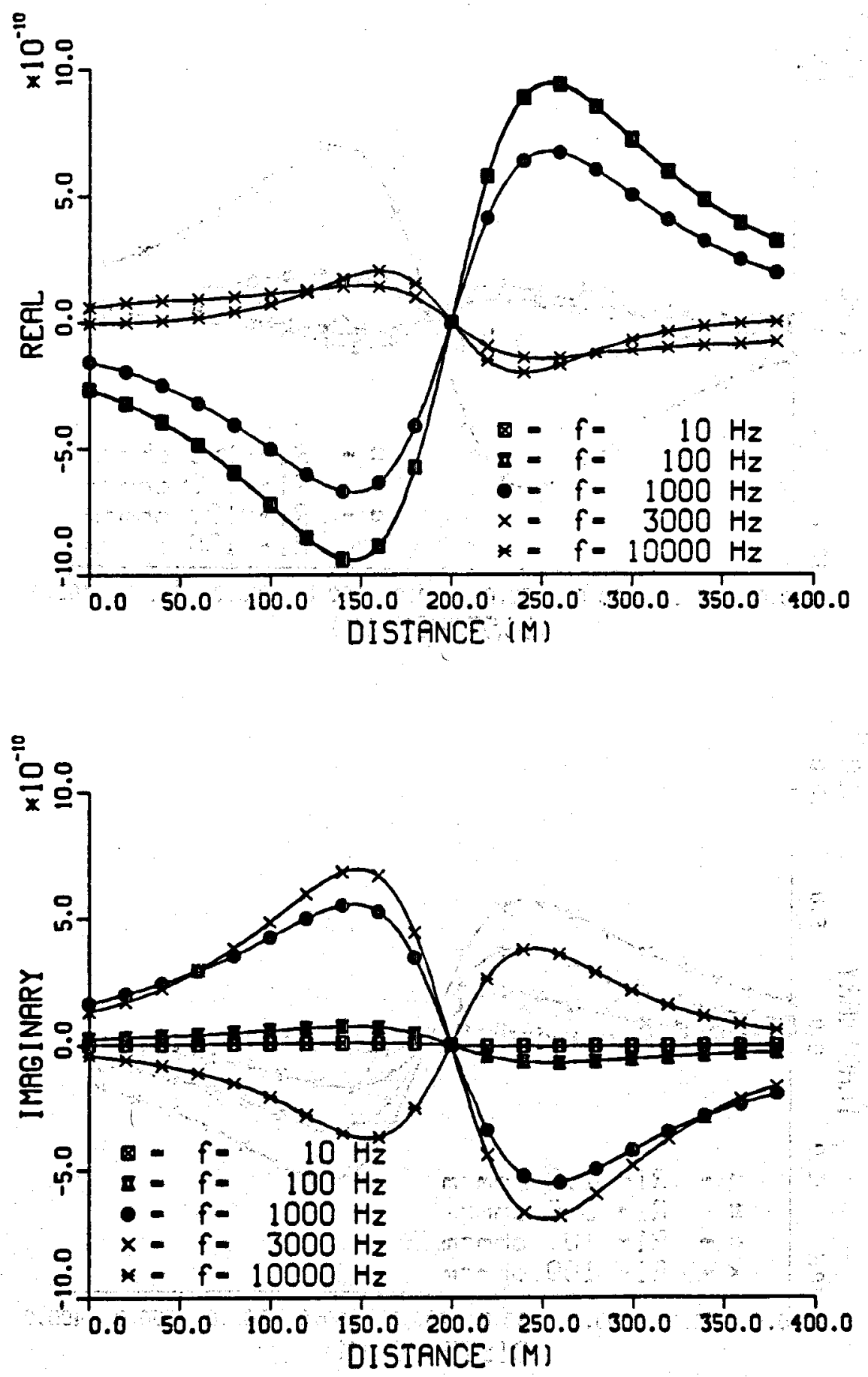

XBL 869-3571

Figure 3. The magnetic field $\left(H_{y}\right)$ anomaly for varying frequency; $f=10,100$, $1000,3000,10000 \mathrm{~Hz}$. The sheet is vertical $\left(\beta=90^{\circ}\right)$, and is buried in a uniform half-space of resistivity $\rho_{1}=\rho_{2}=100 \mathrm{ohm}-\mathrm{m}$. The sheet conductance used is $1.0 \mathrm{~S}$. The magnetic field intensity is expressed in $A / m$ $\times 10^{7}$ (SI units). 

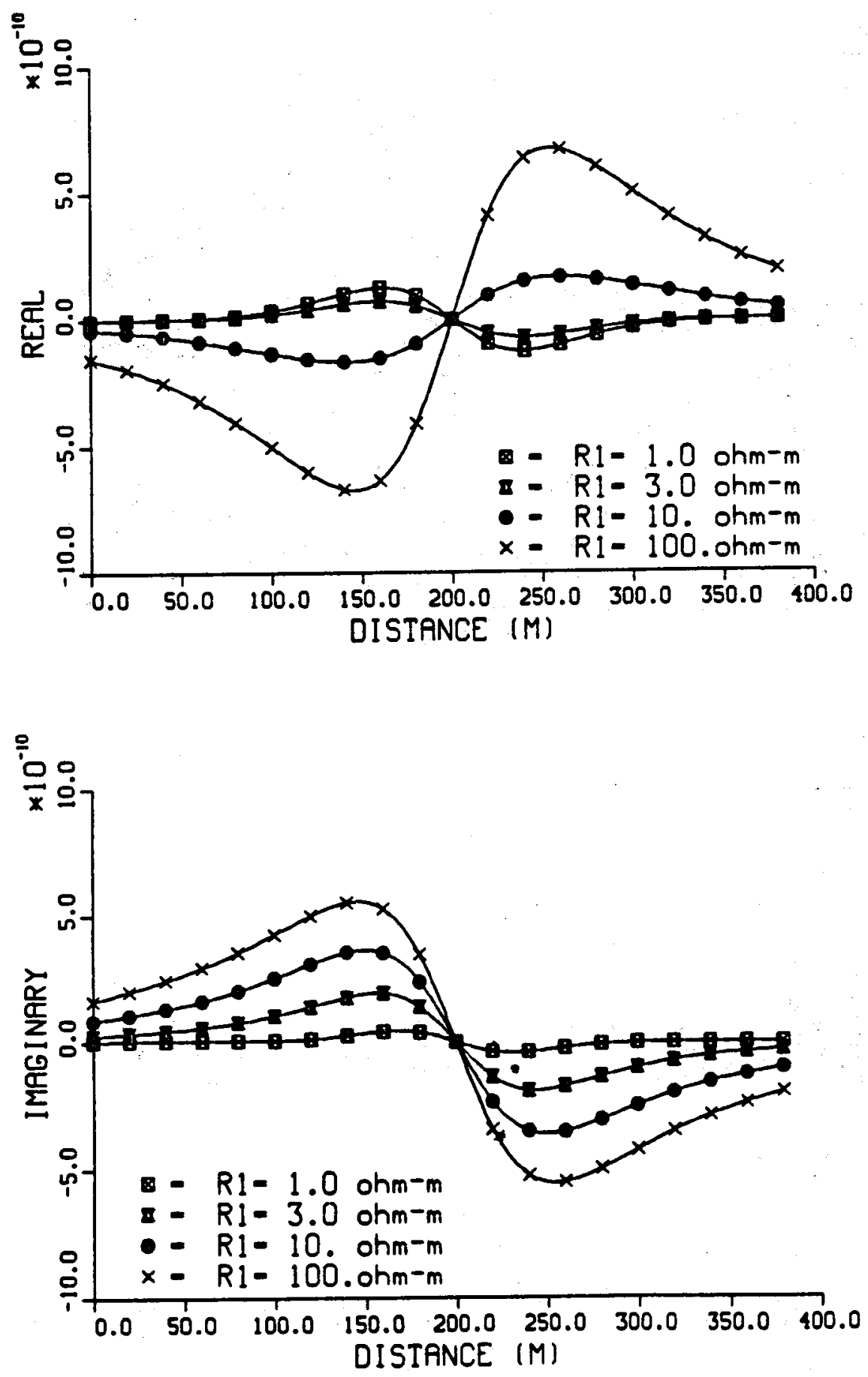

XBL 869-3572

Figure 4. The magnetic field $\left(H_{y}\right)$ anomaly for varying overburden resistivity; $\rho_{1}=$ $1,3,10,100$ ohm-m, with fixed thickness of $D=10 \mathrm{~m}$. The sheet is vertical $\left(\beta=90^{\circ}\right)$, and is buried in the lower half-space of resistivity of $\rho_{2}=100 \mathrm{ohm}-\mathrm{m}$. The sheet conductance used is $1.0 \mathrm{~S}$, and the frequency used is $1000 \mathrm{~Hz}$. The magnetic field intensity is expressed in $\mathrm{A} / \mathrm{m} \times 10^{7}$ (SI units). 

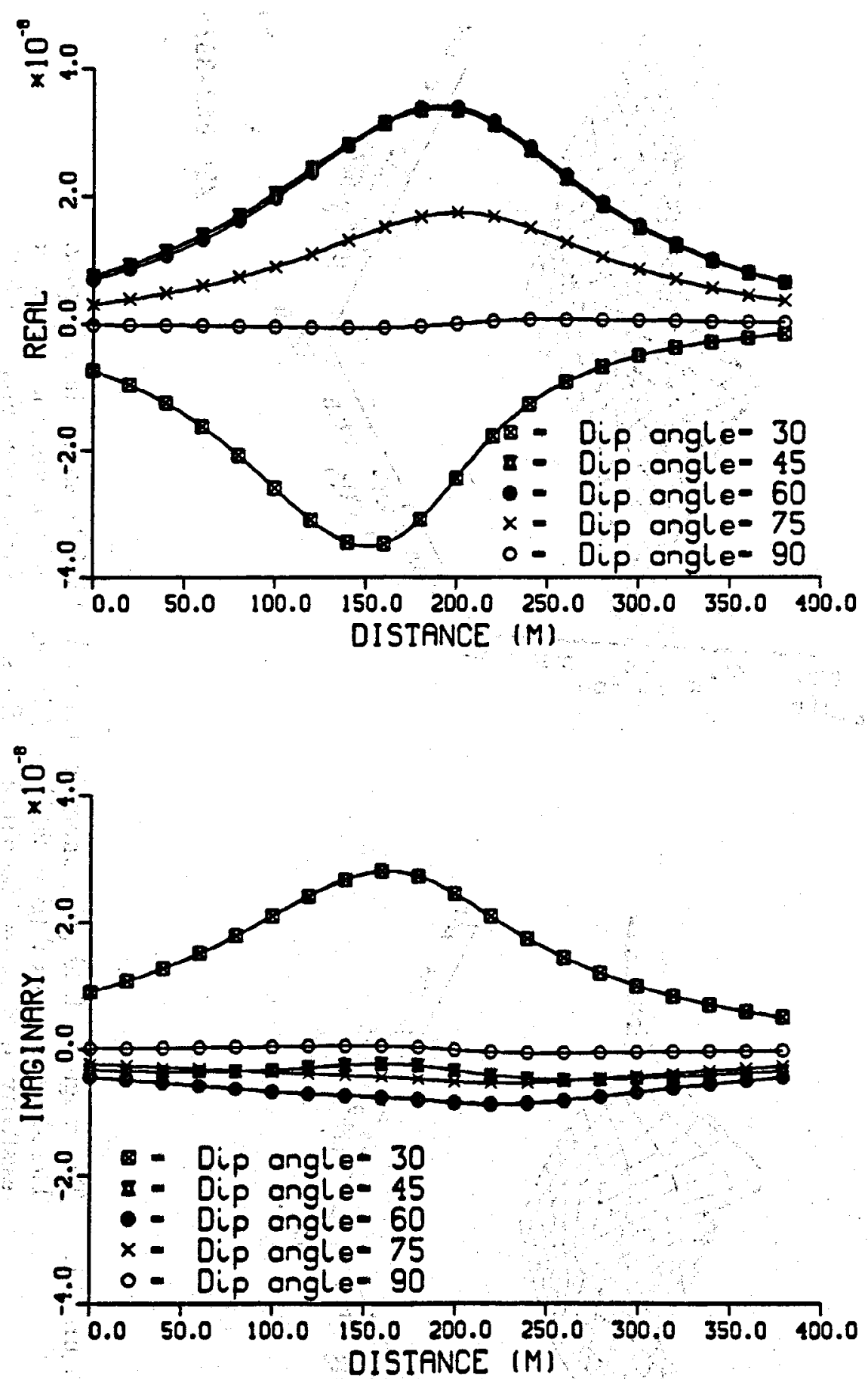

XBL $869-3573$

Figure 5. The magnetic field $\left(H_{y}\right)$ anomaly for varying dip angle; $\beta=30^{\circ}, 45^{\circ}$, $60^{\circ}, 75^{\circ}, 90^{\circ}$. The sheet is buried in a uniform half-space of resistivity $\rho_{1}=\rho_{2}=100 \mathrm{ohm}-\mathrm{m}$. The sheet conductance used is $1.0 \mathrm{~S}$, and the frequency used is $1000 \mathrm{~Hz}$. The magnetic field intensity is expressed in $\mathrm{A} / \mathrm{m}$ $\times 10^{7}$ (SI units). 

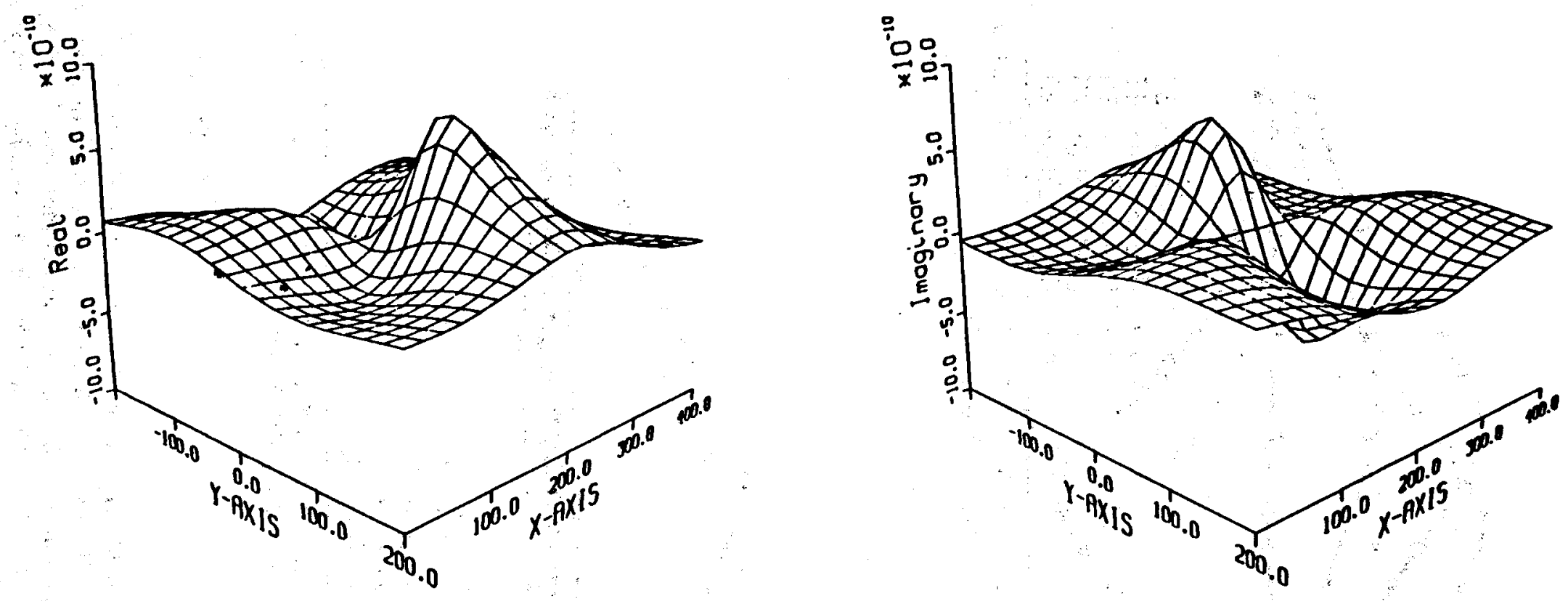

Figure 6.1. The magnetic field $\left(H_{x}\right)$ anomaly due to a G.V.E.D. source in 3-D view. The sheet is vertical $\left(\beta=90^{\circ}\right)$, and is buried in a uniform half-space of resistivity $\rho_{1}=\rho_{2}=100 \mathrm{ohm}-\mathrm{m}$. The sheet conductance used is $1.0 \mathrm{~S}$, and the frequency used is $1000 \mathrm{~Hz}$. The magnetic field intensity is expressed in $\mathrm{A} / \mathrm{m} \times 10^{7}$ (SI units).

XBL 869-3574 


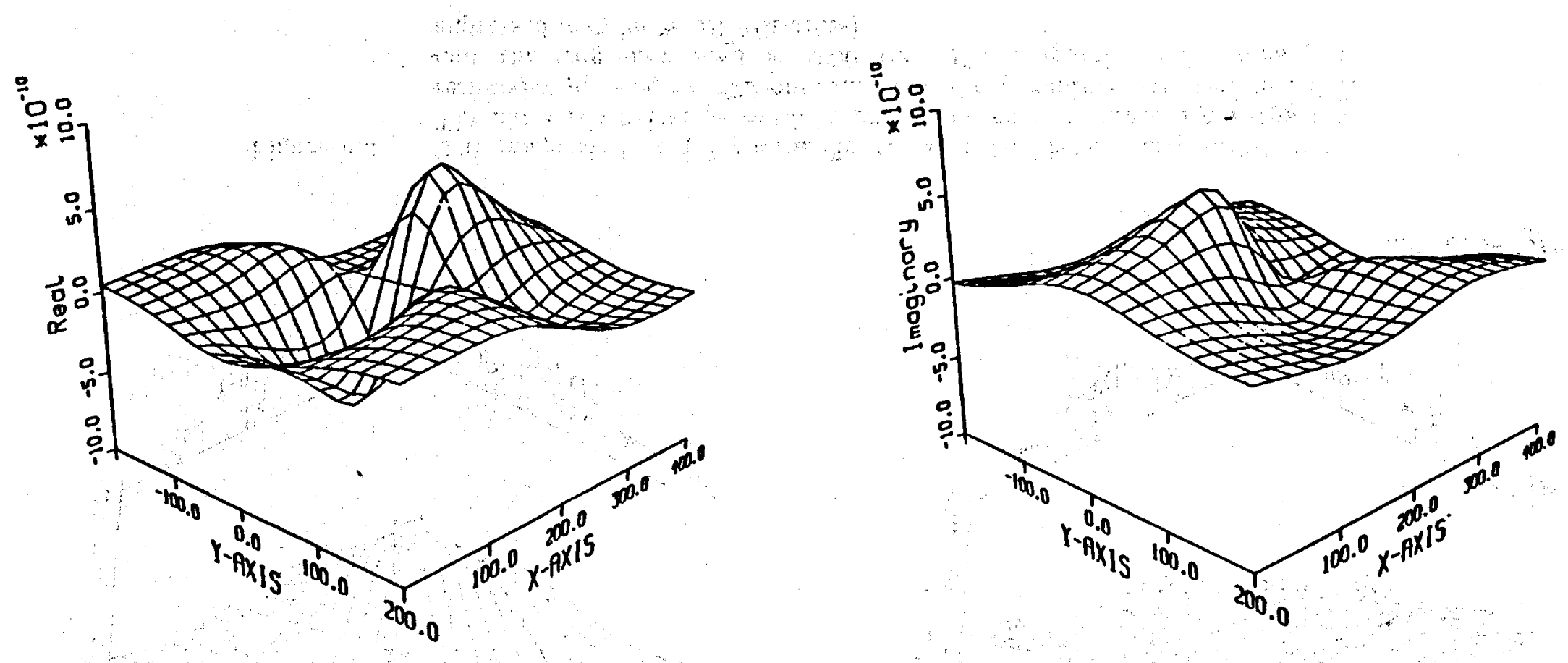

XBL 869-3575

Figure 6.2. The magnetic field $\left(H_{y}\right)$ anomaly due to a G.V.E.D. source in 3-D view. The sheet is vertical $\left(\beta=90^{\circ}\right)$, and is buried in a uniform half-space of resistivity $\rho_{1}=\rho_{2}=100 \mathrm{ohm}-\mathrm{m}$. The sheet conductance used is $1.0 \mathrm{~S}$, and the frequency used is $1000 \mathrm{~Hz}$. The magnetic field intensity is expressed in $\mathrm{A} / \mathrm{m} \times 10^{7}$ (SI units). 

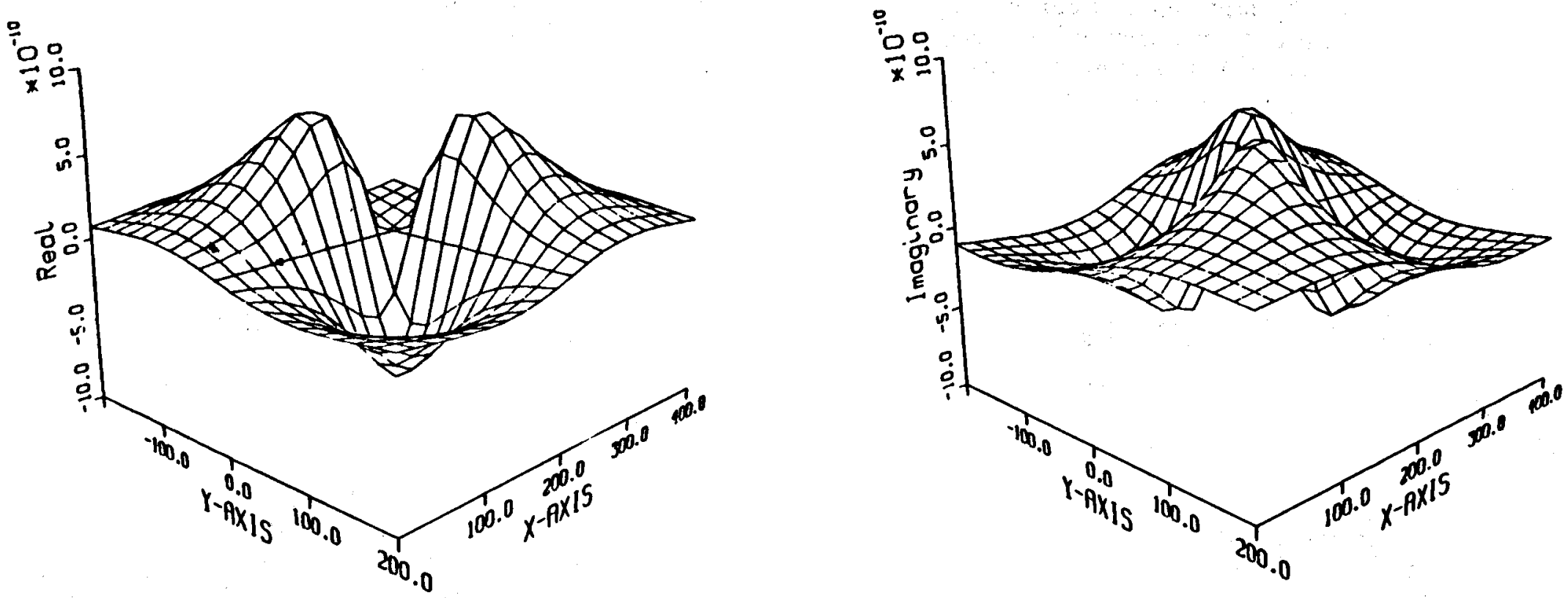

XBL 869-3576

Figure 6.3. The magnetic field $\left(H_{z}\right)$ anomaly due to a G.V.E.D. source in 3-D view. The sheet is vertical $\left(\beta=90^{\circ}\right)$, and is buried in a uniform half-space of resistivity $\rho_{1}=\rho_{2}=100 \mathrm{ohm}-\mathrm{m}$. The sheet conductance used is $1.0 \mathrm{~S}$, and the frequency used is $1000 \mathrm{~Hz}$. The magnetic field intensity is expressed in $\mathrm{A} / \mathrm{m} \times 10^{7}$ (SI units). 

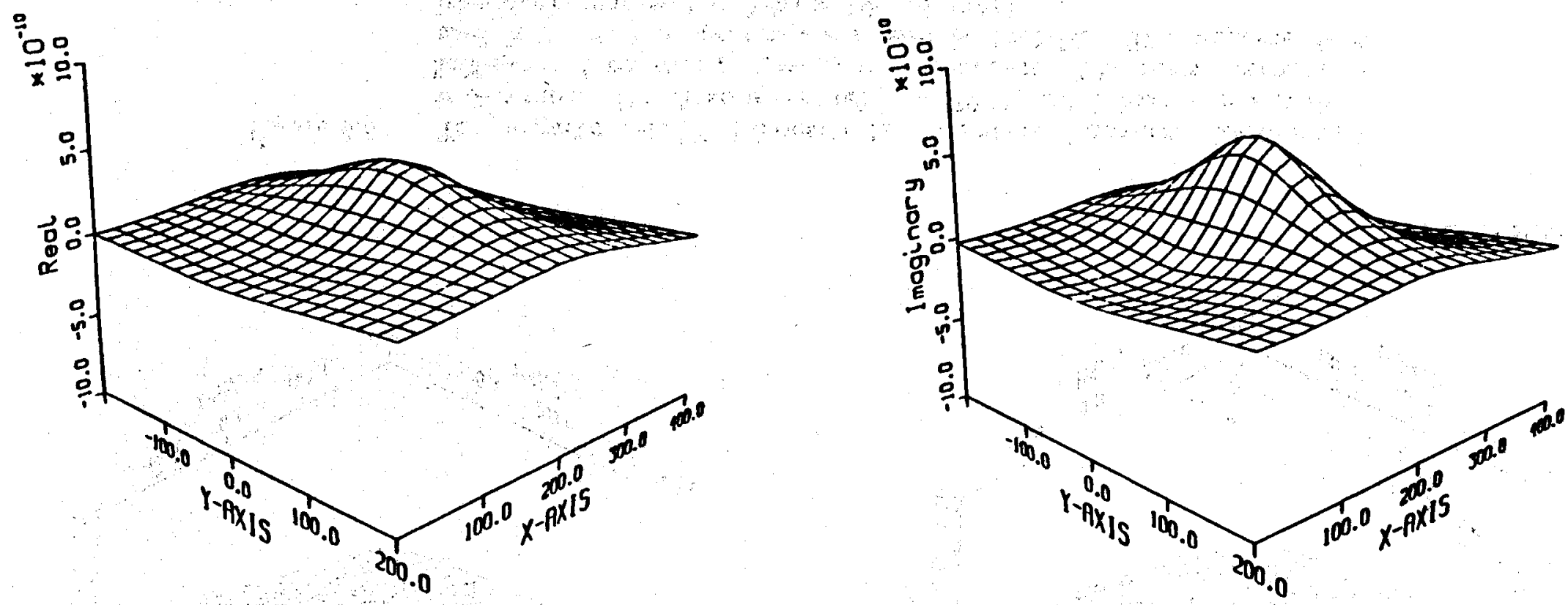

XBL $869-3577$

Figure 6.4. The magnetic field $\left(H_{z}\right)$ anomaly due to a vertical magnetic dipole source in 3-D view. The sheet is vertical $\left(\beta=90^{\circ}\right)$, and is buried in a uniform half-space of resistivity $\rho_{1}=\rho_{2}=100 \mathrm{ohm}-\mathrm{m}$. The sheet conductance used is $1.0 \mathrm{~S}$, and the frequency used is $1000 \mathrm{~Hz}$. The magnetic field intensity is expressed in $\mathrm{A} / \mathrm{m} \times 10^{7}$ (SI units). 

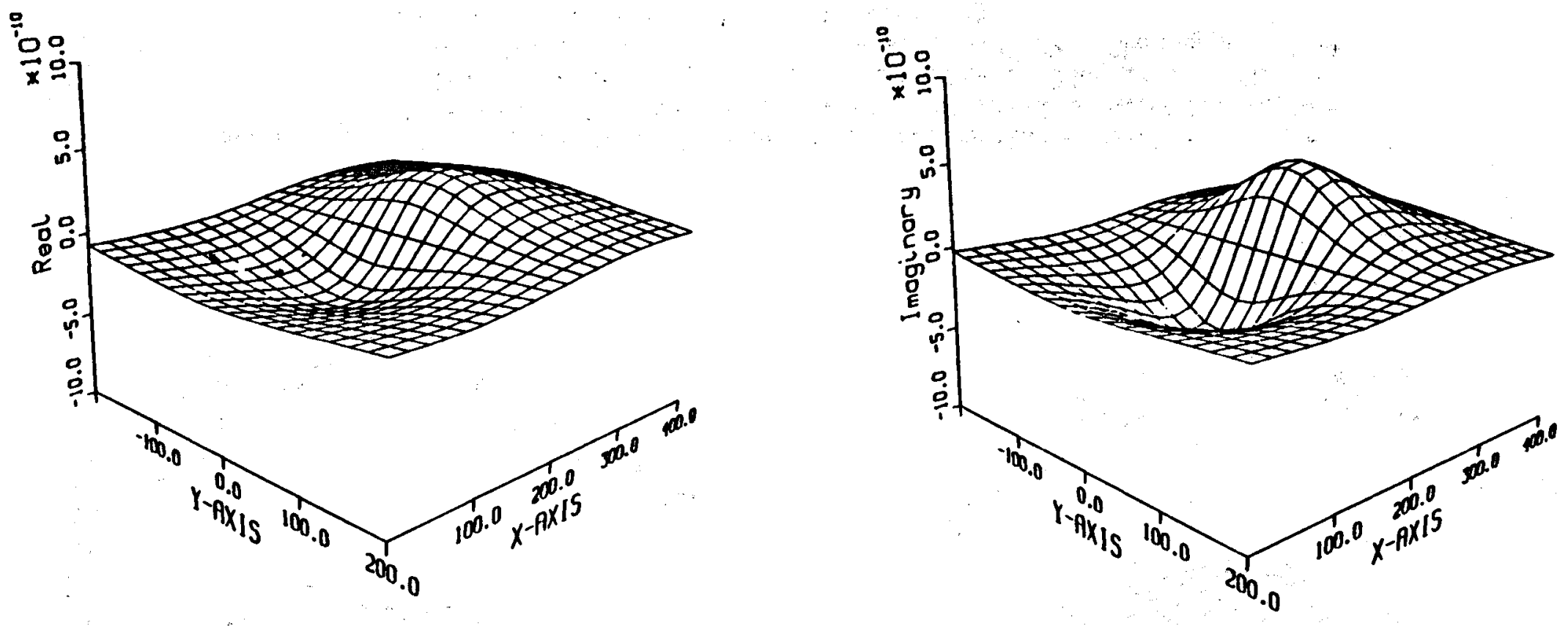

XBL $869-3578$

Figure 6.5. The magnetic field $\left(H_{z}\right)$ anomaly due to a vertical magnetic dipole source in 3-D view. The sheet is vertical $\left(\beta=90^{\circ}\right)$, and is buried in a uniform half-space of resistivity $\rho_{1}=\rho_{2}=100 \mathrm{ohm}-\mathrm{m}$. The sheet conductance used is $1.0 \mathrm{~S}$, and the frequency used is $1000 \mathrm{~Hz}$. The magnetic field intensity is expressed in $\mathrm{A} / \mathrm{m} \times 10^{7}$ (SI units). 
(A) Side View

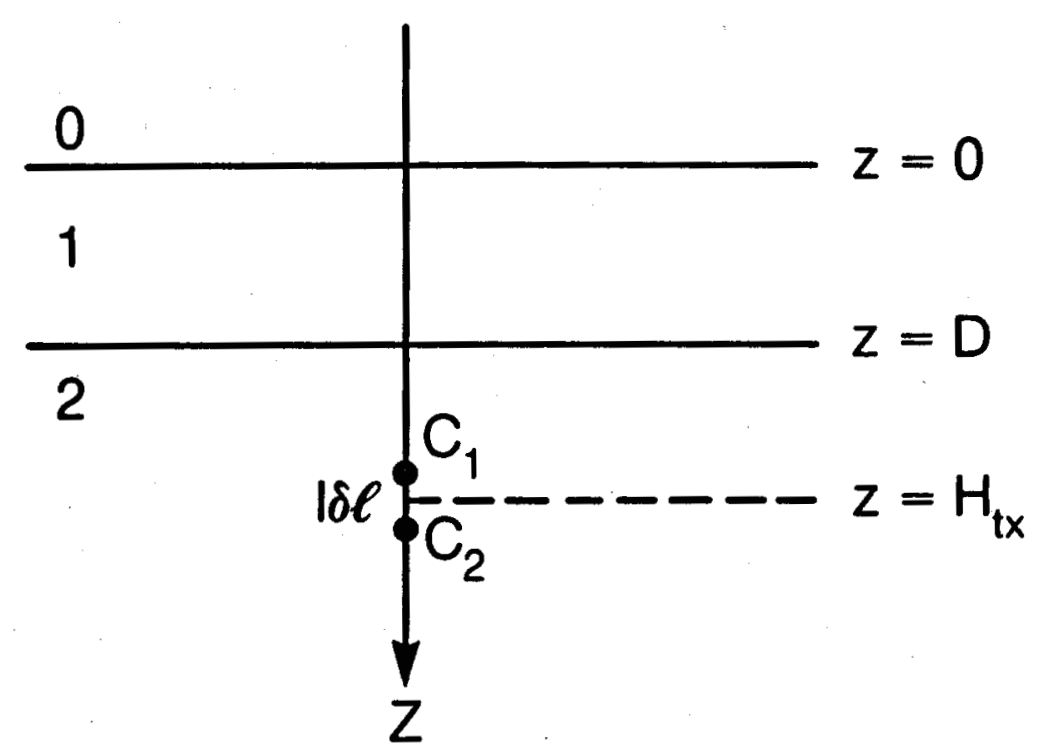

(B) Top View

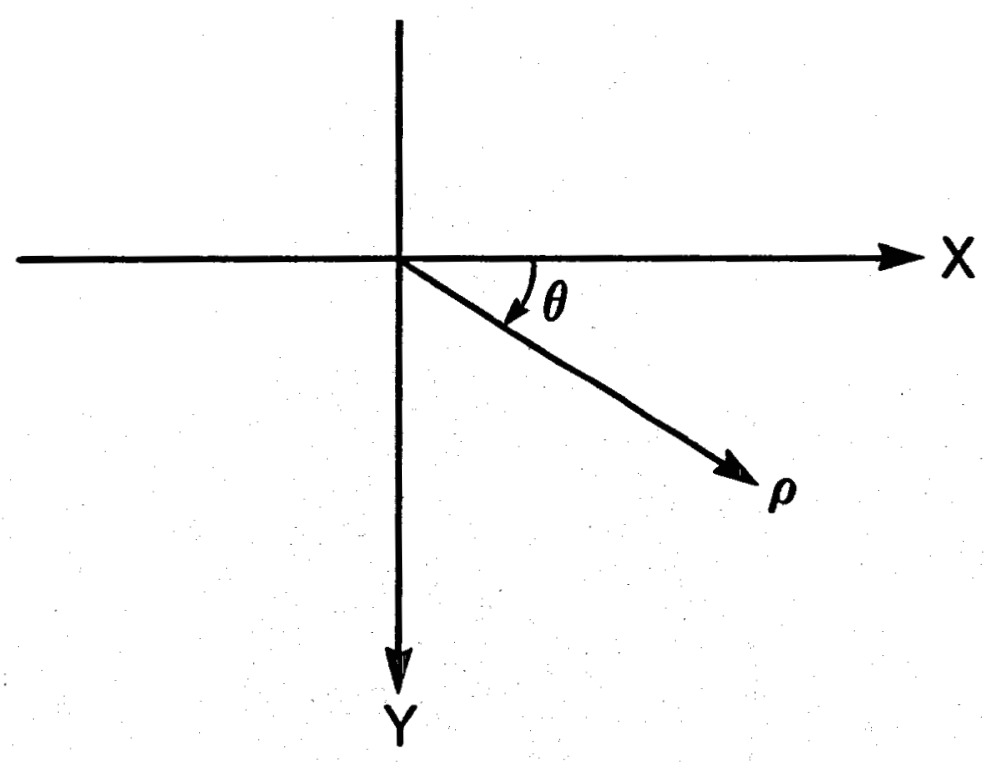

XBL 869-10992

Figure A.1. Two layered earth model used for the calculation of normal electric field $\mathbf{E}_{\mathrm{ns}}$ due to an oscillating electric dipole of moment $I \delta l$ buried at $z=H_{t x}$. 